\title{
DIFFUSIOPHORESIS-DRIVEN STRATIFICATION OF POLYMERS IN COLLOIDAL FILMS
}

\author{
Malin Schulz ${ }^{\dagger,},{ }^{*}$ Richard W. Smith ${ }^{\ddagger}$, Richard P. Sear ${ }^{\dagger}$, Richard Brinkhuis ${ }^{\S}$, Joseph L. Keddie ${ }^{\dagger, *}$
}

$\uparrow$ Department of Physics, University of Surrey, Guildford, Surrey, GU2 7XH, United Kingdom † Surrey Ion Beam Centre, University of Surrey, Guildford, Surrey, GU2 7XH, United Kingdom

$\S$ Allnex, Nieuwe Kanaal 7N, 6709 PA Wageningen, The Netherlands

* E-mail: $\underline{\text { m.schulz@ @urrey.ac.uk }}$

* E-mail: j.keddie@ surrey.ac.uk

\section{EXPERIMENTAL METHODS FOR FILM FORMATION}

To control the temperature of the samples during film formation, they were placed on a heating stage (Linkam TP93, Tadworth, UK). Wet films were cast on a Si (100) substrate by depositing $380 \mu \mathrm{L}$ using a micropipette and spreading to cover the entire area of the substrate $(2 \mathrm{~cm} \times 2 \mathrm{~cm})$ to make a wet film on the order of $1 \mathrm{~mm}$ thick. Samples were placed onto the stage pre-heated to the setpoint temperature. The silicon substrates were pre-treated using a UV ozone cleaner (Bioforce Nanosciences Inc) to remove any organic contaminants. Using an IR camera (FLIR E60 bx) to measure the temperature of the wet film, we confirmed that there were no significant temperature gradients laterally in the film plane and that the films reached the target temperature within a short time.

The heating stage was placed within a glovebox that was equilibrated at a target relative humidity $(\mathrm{RH})$ using silica gel $(\mathrm{RH}=20 \%)$, ambient air $(\mathrm{RH}=42 \%)$, or a saturated sodium chloride solution $(\mathrm{RH}=70 \%)$. We monitored the relative humidity in the glovebox using an Oregon Scientific THGR810 hygrometer. In some experiments, the sample was left in the ambient atmosphere $(\mathrm{RH} \approx 50 \%)$ and flowing air from an electric rotary fan was directed over the sample.

To measure water evaporation rates under the range of environmental conditions, film were cast on glass plates and placed on a digital balance (Sartorius Entris 224i-1s). The mass of the wet film, $m$, was recorded over the first five min. of drying in the region where the data are linear. The gradient of the data, $\Delta m / \Delta t$, was determined. Knowing the density of the evaporating water, $\rho=$ $1.0 \mathrm{~g} \mathrm{~cm}^{-3}$ and the surface area of the drying film, $A$, we calculated the evaporation rate (in units of velocity), $E$, with this equation:

$$
E=\frac{-\left(\frac{\Delta m}{\Delta t}\right)}{A \cdot \rho}
$$




\section{CALCULATION OF PÉCLET NUMBERS}

The Péclet number for each of the experiments was calculated as:

$$
P e=\frac{H E}{D}
$$

where $H$ is the initial wet film thickness and $D$ is the diffusion coefficient of the polymer in solution. As the solutions were initially in dilute limit $\left(\phi<\phi^{*}\right)$, the Stokes-Einstein equation was used to calculate the self-diffusion coefficient, for a swollen polymer coil with a hydrodynamic radius, $R_{h}$, as:

$$
D=\frac{k_{B} T}{6 \pi \eta R_{h}}
$$

where $k_{\mathrm{B}}$ is the Boltzmann constant, $T$ is the absolute temperature, and $\eta$ is the viscosity of the continuous liquid phase. The $R_{h}$ of poly(acrylic acid) in water was taken from the literature ${ }^{1}$ to be $14.5 \mathrm{~nm}$. Values for the viscosity of water as a function of temperature were also obtained from the literature. ${ }^{2}$

Through control of the volume of the wet dispersion, we set $H$ to be $1 \mathrm{~mm}$ in all experiments. In Table S1 we show data for the dPAA and the resulting Pe.

Table S1. Parameters of the Experiments and Calculation of the Péclet Numbers*

\begin{tabular}{|l|l|l|ll|l|l|}
\hline$T\left[{ }^{\circ} \mathrm{C}\right]$ & $\begin{array}{l}\text { Relative } \\
\text { humidity } \\
{[\%]}\end{array}$ & $\eta[\mathrm{mPas}]$ & $\begin{array}{c}D \\
\left.\mathrm{~m}^{2} / \mathrm{s}\right]\end{array}$ & {$\left[10^{-11}\right.$} & $E\left[\frac{\mathrm{nm}}{\mathrm{s}}\right]$ & $\mathrm{Pe}$ \\
\hline 60 & $\mathrm{FA}^{\S}$ & 0.470 & 3.58 & 555 & 24.5 \\
40 & $\mathrm{FA}^{\S}$ & 0.656 & 2.33 & 382 & 25.0 \\
40 & 42 & 0.656 & 2.33 & 151 & 9.9 \\
30 & 42 & 0.801 & 1.97 & 93 & 7.7 \\
20 & 20 & 1.004 & 1.48 & 22 & 2.3 \\
20 & 70 & 1.004 & 1.48 & 10 & 0.7 \\
\hline
\end{tabular}

${ }^{*} H=0.001 \mathrm{~m}$ and $R_{\mathrm{h}}=14.5 \mathrm{~nm}$ for all experiments. $\S \mathrm{FA}=$ flowing air.

\section{SURFACE CHARACTERIZATION METHODS: AFM AND WATER CONTACT ANGLE.}

We used an atomic force microscope (Bruker Dimension Edge with Scan Asyst) to examine the surface structures. AFM was conducted with intermittent contact using an antimony n-doped Si cantilever with a nominal spring constant of $42 \mathrm{~N} / \mathrm{m}$ (Bruker NCHV). We measured the contact angle of water on exactly the same samples analyzed by ERD, using a commercial apparatus (Krüss DSA 25B). In each measurement, $5 \mu \mathrm{L}$ sessile droplets of water were used to determine the static contact angle via image analysis. At least three measurements each at different positions on the sample surfaces were made to find the mean value. Advancing and receding contact angle measurements were made using the same apparatus. 


\section{ESTIMATE OF THE DIFFUSIOPHORETIC SPEED}

The diffusiophoretic speed of our colloids, $U$, is from Equation (4) of Sear and Warren ${ }^{3}$

$$
U \cong D \frac{\partial \phi_{p p}(z)}{\partial z}
$$

The diffusiophoretic coefficient that relates the diffusiophoretic speed of the larger colloid to the concentration gradient of the smaller polymer coil is approximately just the diffusion constant $D$ of the polymer.

In the evaporating liquid film, the concentration gradient of the polymer from Equation (15) of Sear and Warren is

$$
\frac{\partial \phi_{p p}}{\partial z} \cong \frac{E P e \phi_{p p}}{D}
$$

This is the maximum value of the gradient, which is found just below the descending air/water interface. If we combine these two equations, we have the very simple result

$$
U \cong E P e \phi_{p p}
$$

This is the diffusiophoretic speed of the colloid, just below the air/water interface. Just below the interface, the diffusiophoretic speed of the colloids exceeds the evaporation speed whenever $P e \phi_{p p}>1$.

As an example, for the second system in Table $1, P e=25$. In most of our experiments, including this one, $\phi_{p p}=0.2$. Hence, the maximum diffusiophoretic speed is approximately five times $E=0.4 \mu \mathrm{m} \mathrm{s}^{-1}$, or about $2 \mu \mathrm{m} \mathrm{s}^{-1}$. 


\section{ENERGY CALIBRATION AND SOLID ANGLE DETERMINATION FOR ION BEAM ANALYSIS}

We performed the ion beam analysis (IBA) experiments in the broad beam chamber of the University of Surrey $2 \mathrm{MV}$ Tandetron particle accelerator using a 1-2 mm diameter ${ }^{4} \mathrm{He}^{+}$beam. We used a Si PIN photodiode as a particle detector in IBM geometry to acquire ERD spectra and a PIPS particle detector in Cornell geometry to acquire RBS spectra. The detector and beam setup can be seen in Figure S1. There is a range foil in front of the ERD detector to stop ${ }^{4} \mathrm{He}^{+}$ions. However, there is still an increase in counts at low energies because some ${ }^{4} \mathrm{He}^{+}$ions pass through the foil.

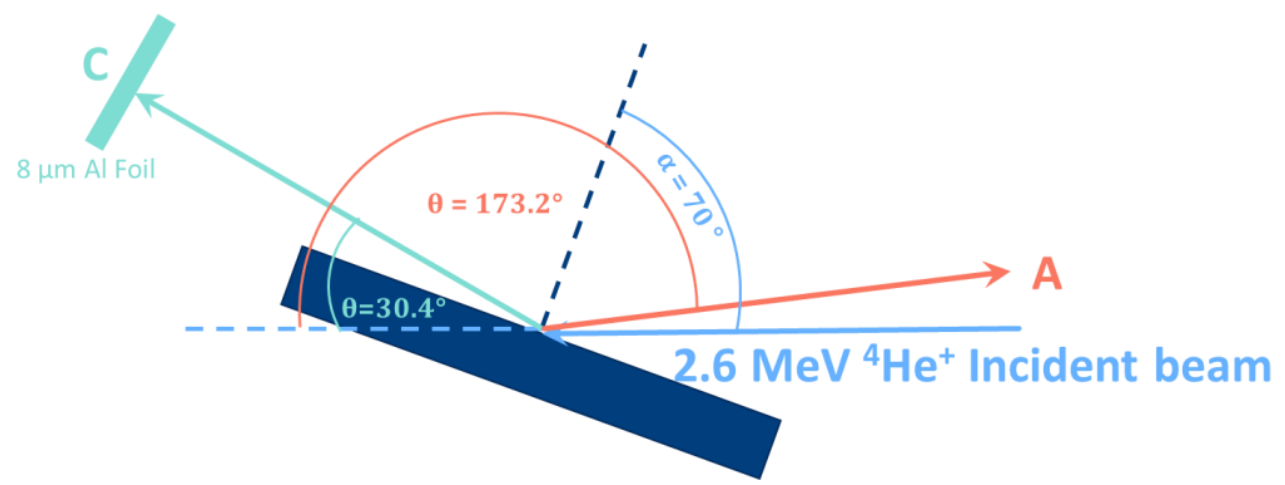

Figure S1. Detector and beam setup of the IBA experiments performed. The beam is incident on the sample at an angle of $70^{\circ}$ to the sample normal. Detector A is used for RBS and is in the Cornell geometry. Detector $C$ is used for ERD and is in the IBM geometry. In front of detector $C$ is an $8 \mu \mathrm{m}$ thick Al range foil.

To determine the energy calibration of detector C (ERD), we took spectra from thin films of hydrogenated poly(styrene) (hPS) and d8 deuterium-labelled poly(styrene) (dPS) (from Polymer Laboratories, Church Stretton, UK), spin-cast from toluene onto (100) Si substrates. We used the SIMNRA software ${ }^{4}$ to model the data. We used the SigmaCalc 2.0 recoil cross-section ${ }^{5}$ for hydrogen and the Kellock and Baglin recoil cross-section ${ }^{6}$ for deuterium. We used an estimate of the number of particles-solid angle product, $N \Omega$ (as is required by SIMNRA). This parameter, $N \Omega$, is simply calculated as the product of the solid angle subtended by the detector at the sample $(\Omega)$ and the number of particles incident on the sample $(N)$. The number of particles can be calculated by dividing the obtained live charge $\left(Q_{\text {live }}\right)$ by the charge on one ion, in this case $1.6 \times 10^{-19} \mathrm{C}$.

We then performed simulations on both calibration samples in turn. We adjusted the energy per channel, calibration offset, and detector resolution until there was a good agreement between the simulation and experimental data for both samples' spectra. The calibrated parameters we determined are:

Energy per channel: $0.735 \mathrm{keV} / \mathrm{ch}$

Calibration offset: $46 \mathrm{keV}$

Detector resolution: $75 \mathrm{keV}$

We used these parameters for analysing data from all subsequent experiments.

To determine the solid angle, $\Omega$, we used a $2.2 \mu \mathrm{m}$ thick hPS film on Si. In a similar way as with the samples presented in the main paper, we obtained spectra in sets of $2 \mu \mathrm{C}$ charge. We used the same energy calibration and detector resolution as determined with the standard samples. 
We fitted each spectrum by adjusting $N \Omega$ until we obtained good agreement between the simulation and experimental data. We calculated the solid angle by dividing $N \Omega$ by the number of particles obtained from the live charge. With increasing accumulated charge, $Q_{a c c}$, the solid angle $\Omega$ appeared to decrease. However, in reality, $\Omega$ is a constant but the number of recoiled hydrogen atoms detected is decreasing, hence changing the $N \Omega$ product. This effect results from the change in the sample composition (i.e. loss of hydrogen) caused by beam-induced heating. To correct for this effect, we plotted the calculated solid angles against the respective accumulated charge, as can be seen in Figure S2. After fitting a linear regression, we estimated $\Omega$ at an accumulated charge of $Q_{a c c}=0 \mu \mathrm{C}$, i.e. without any loss of hydrogen, to be $0.396 \mathrm{msr}$. The RBS detector A was calibrated with the same method.

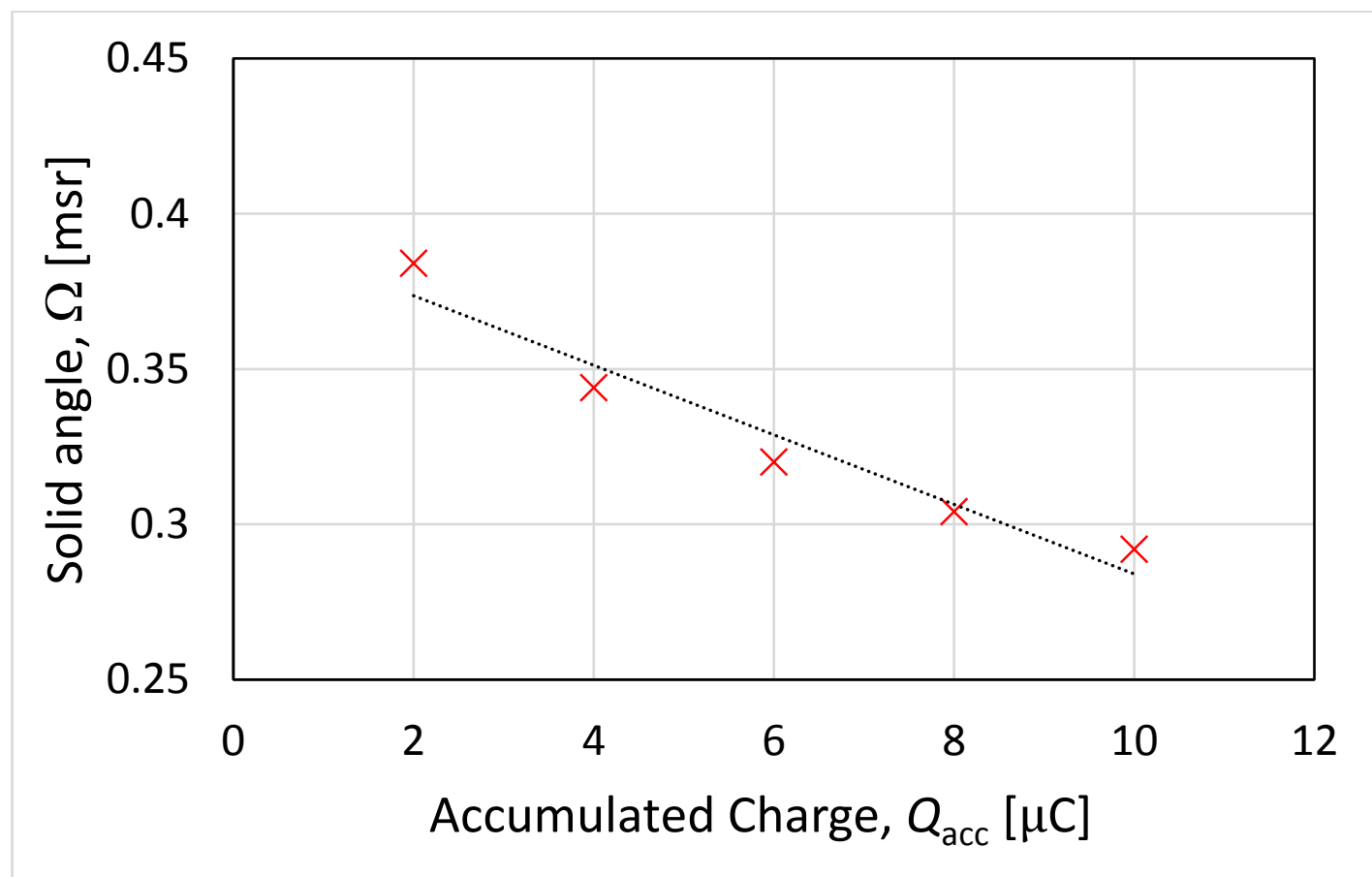

Figure S2. Calculated solid angle, $\Omega$, plotted against the accumulated charge, $Q_{\text {acc, }}$ for each spectrum. The red crosses show the experimental data, and the dotted line is a linear fit given by $\Omega=-0.0112 Q_{a c c}+0.396$.

\section{EVALUATION OF BEAM-INDUCED DAMAGE}

Due to the high beam energy of $2.6 \mathrm{MeV}$, the samples suffer from beam-induced heating and partially carbonize during the measurement. After the ion beam experiments, samples exhibit a black mark on the surface, which is visible by eye. Raman spectroscopy (Renishaw inVia) was used to confirm the existence of elemental amorphous $\mathrm{C}$ in the PAA/latex films. After an exposure of $10 \mu \mathrm{C}$, the sample spectrum from a black mark shows the D- and G-bands of amorphous $\mathrm{sp}^{2}$ carbon at $1352 \mathrm{~cm}^{-1}$ and $1591 \mathrm{~cm}^{-1}$, respectively. ${ }^{7,8}$ Figure S3 shows the spectra obtained from the sample surface before and after the ion beam exposure. It is apparent that there has been beaminduced carbonization of the copolymer. 


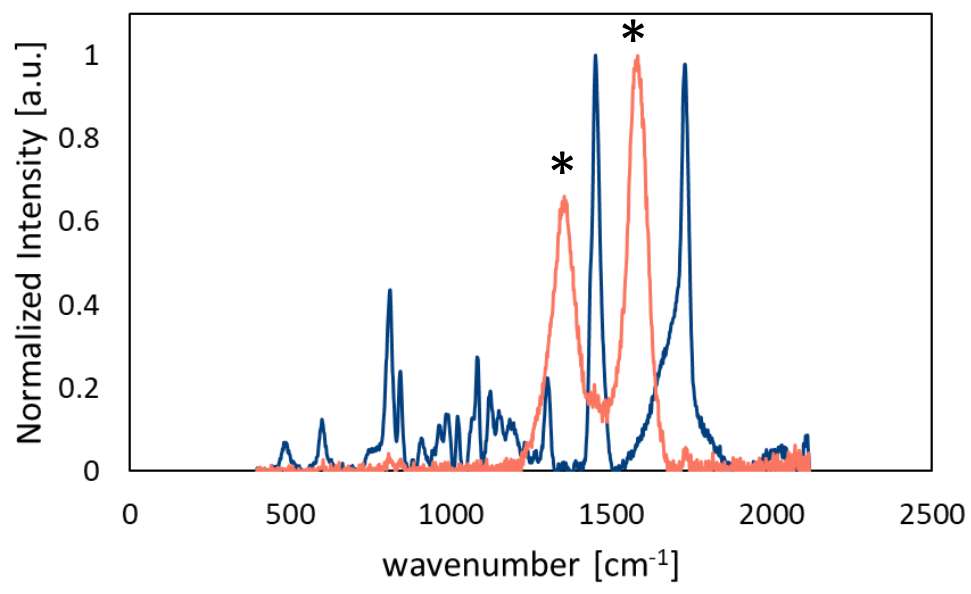

Figure S3. Raman spectra of a sample containing a mixture of linear poly(acrylic acid) and the acrylic latex before (blue) and after (orange) the ion beam analysis. In the "after" spectrum (orange), there are two strong signals attributed to $\mathrm{sp}^{2}$ amorphous carbon (at $1352 \mathrm{~cm}^{-1}$ and 1591 $\mathrm{cm}^{-1}$ ) marked with * and only very weak signals from the original polyacrylate copolymer.

Exposure to the ion beam leads to a loss of both hydrogen and deuterium and to an alteration of the sample composition. The change in composition during a measurement is shown in Figure S4 where we plot the individual $2 \mu \mathrm{C}$ spectra for measurements of two dPAA/latex film samples, film-formed with different Pe. As we can see in Figure S4a for a sample with $\mathrm{Pe}=24.5$, the counts in the first $2 \mu \mathrm{C}$ spectrum is lower than in the second $2 \mu \mathrm{C}$ spectrum. This phenomenon was seen in some samples, and we do not have an explanation for it. In Figure S4b we see a second example (with $\mathrm{Pe}=25$ ) where the counts decrease with increasing accumulated charge, as is expected if there is a build-up of carbon at the sample surface. It is notable that regardless of whether the first spectrum has lower or higher counts than the following spectra, the ratios between deuterium and hydrogen peaks stay approximately the same. Hence, our comparison of the amounts of dPAA enrichment near the surface of the various samples is valid.

(a)

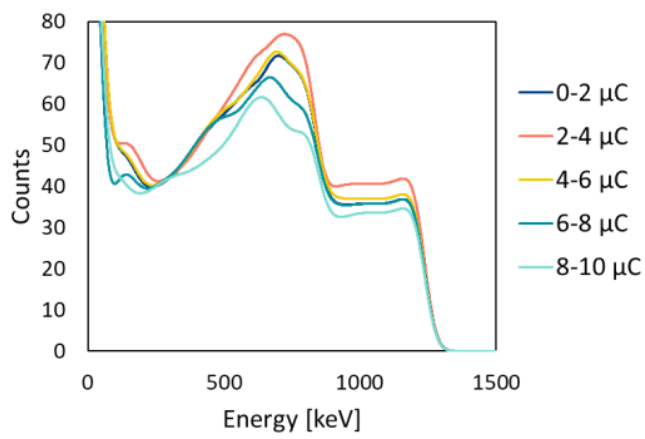

(b)

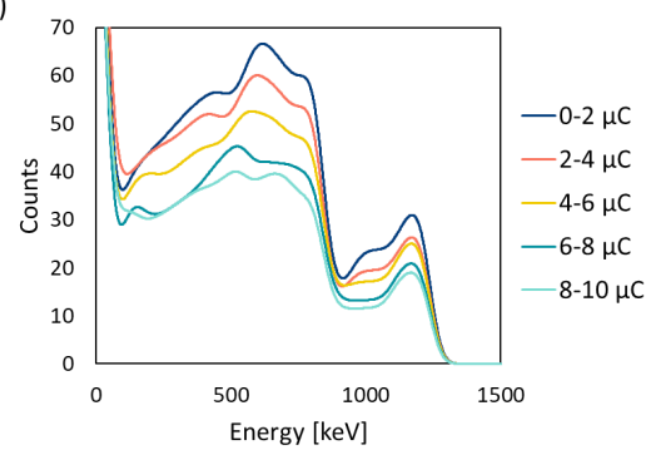

Figure S4. ERD data shown as the individual $2 \mu \mathrm{C}$ spectra for two different samples: (a) Dried at $60^{\circ} \mathrm{C}$ with $\mathrm{Pe}=24.5$ and (b) dried at $40^{\circ} \mathrm{C}$ with $\mathrm{Pe}=25.0$. The accumulated charge, $Q_{\text {acc }}$, for each spectrum is shown in the legend. In (a) the first spectrum appears to be lower in counts than the second and the third. In (b) the number of counts decreases progressively with a higher $Q_{\text {acc. }}$

For accurate simulation of the data, we took the beam-induced damage into account. As the hydrogen and deuterium peaks overlap because of the high sample thickness, it is difficult to judge the loss of hydrogen based on the decreasing height of the hydrogen peak. Therefore, we estimated only the deuterium loss and assumed that hydrogen was lost at a similar rate.

To determine the deuterium loss as charge was accumulated, we summed the counts in the energy range from 1080 to $1250 \mathrm{keV}$ for all five $2 \mu \mathrm{C}$ spectra. Plotting these sums normalized by 
the corresponding live charge $\left(Q_{\text {live }}\right)$ against the accumulated charge $\left(Q_{\text {acc }}\right)$, we fitted a weak exponential function to the data points, as can be seen in Figure S5. Using the fit to extrapolate back to $0 \mu \mathrm{C}$ charge, we calculated the \% loss of deuterium after each $2 \mu \mathrm{C}$ of accumulated charge. From these data we then took the average \% loss, as the total $10 \mu \mathrm{C}$ spectrum is essentially showing a spectrum with an average beam-induced damage. The calculated average deuterium loss for the sample shown in Figure S5 was 24\%. Considering all of the samples in the experiments, the average deuterium loss was $23.6 \pm 11 \%$.

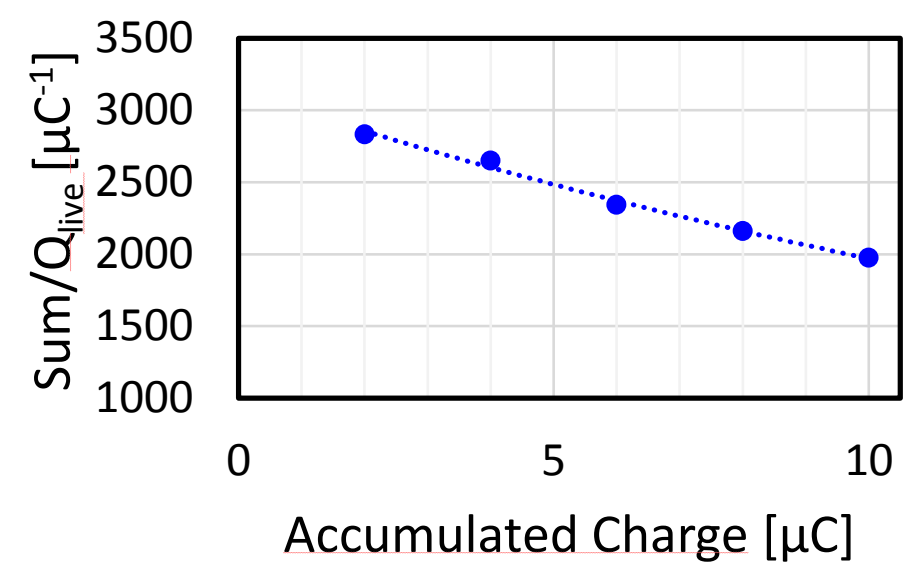

Figure S5. Plot showing the sum of deuterium counts in the range from 1080 to $1250 \mathrm{keV}$ normalized by the live charge $\left(Q_{\text {live }}\right)$ for each five $2 \mu \mathrm{C}$ spectra against the corresponding accumulated charge, $Q_{\text {acc }}$. The solid blue circles show the individual data points. The dotted line shows the exponential function that was fitted to the data as: $\mathrm{Sum} / Q_{\text {live }}=3133.3 \exp \left(-0.046 Q_{\text {acc }}\right)$ with $\mathrm{R}^{2}=0.9937$. On average, the sample shown here lost $24 \%$ of its deuterium during the ERD analysis. 


\section{ION BEAM DATA FOR FILMS WITH $\phi p \mathrm{p}=0.2$ AND WITH VARYING PE}

Looking at the raw data for a range of samples, we can clearly see a trend in the deuterium depth distribution with the variation of the drying conditions. Figure S6 compares four ERD spectra obtained from samples formed at increasing temperatures and hence increasing $E$ and Pe. The number of counts in the energy range above the $\mathrm{H}$ edge at channel 1200 increases with increasing film formation temperatures. It is immediately apparent that more deuterium is accumulating near the surfaces of the films.

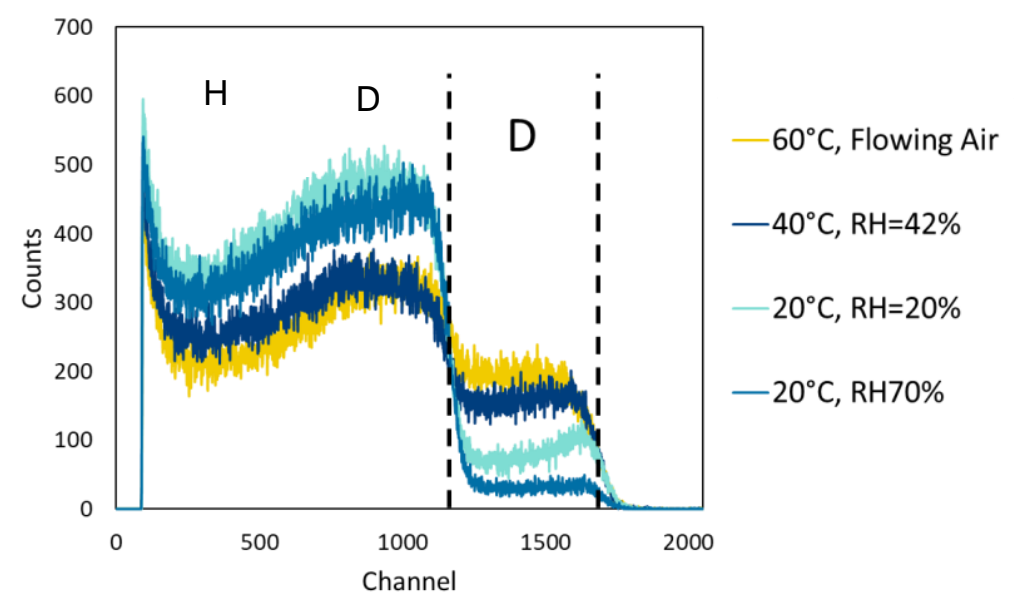

Figure S6. Raw data from ERD experiments for four samples (all with $\phi_{\mathrm{pp}}=0.2$ ) with increasing film formation temperatures, as are shown in the legend. In total, $10 \mu \mathrm{C}$ of charge was accumulated. 


\section{FITTED ERD AND RBS DATA}

Using the SIMNRA software, the RBS spectra were fitted to the same model used for the ERD spectra that were obtained simultaneously. Fitting the RBS data confirmed the $\mathrm{C}$ and $\mathrm{O}$ compositions. The same sample compositions were found to fit both the ERD spectra and the RBS spectra equally well.
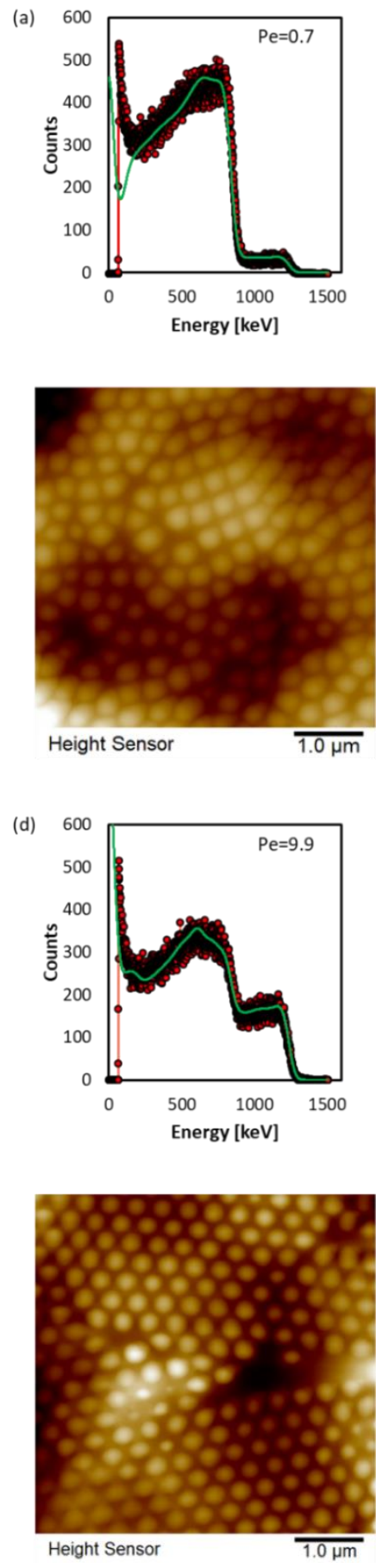
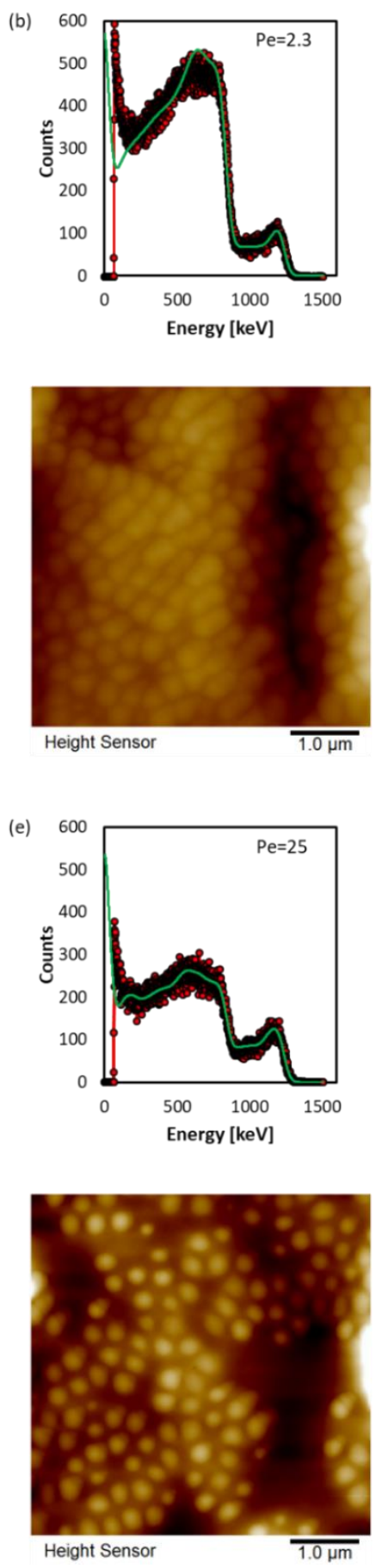
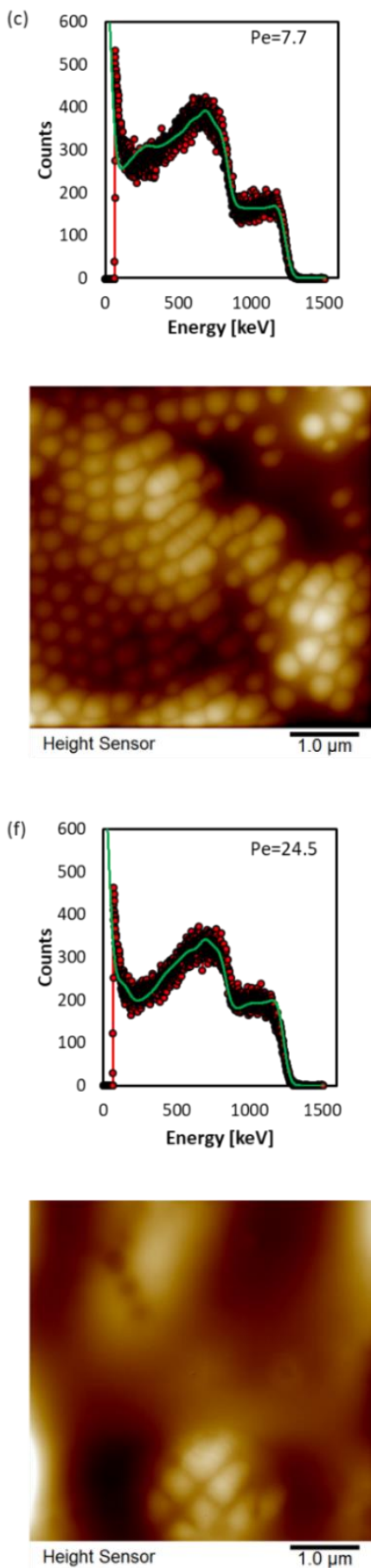

Figure S7. Elastic recoil detection data and corresponding AFM topographic images for the same dPAA/latex films. (a-f) The top rows show the ERD raw data (red circles) and the best fits to the data (green line). The second rows show the AFM topographic images. The Péclet numbers of the samples are $0.7,2.3,7.7,9.9,25$ and 24.5 from (a) to (f). For all samples, the volume fractions were fixed to $\phi_{\mathrm{pp}}=0.2$ and $\phi=0.015$ for the colloid. 


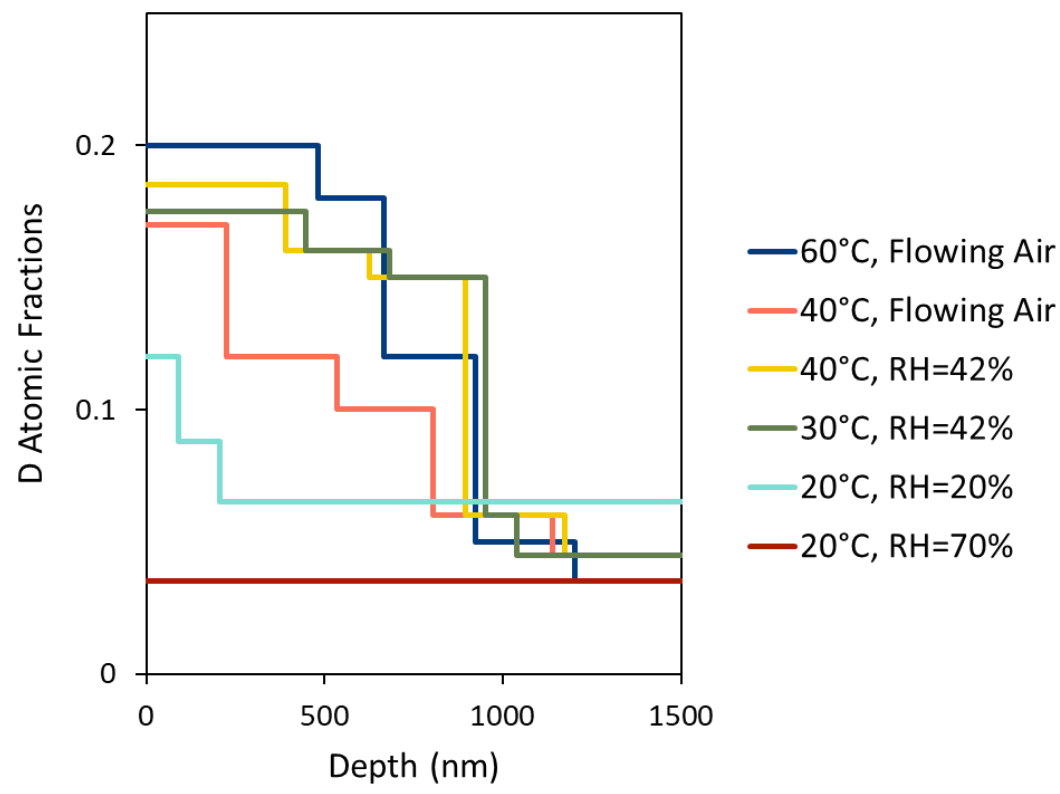

Figure S8. Deuterium depth profiles obtained from analysis of the ERD spectra in Figure S7. The temperature of film formation is represented in the legend.

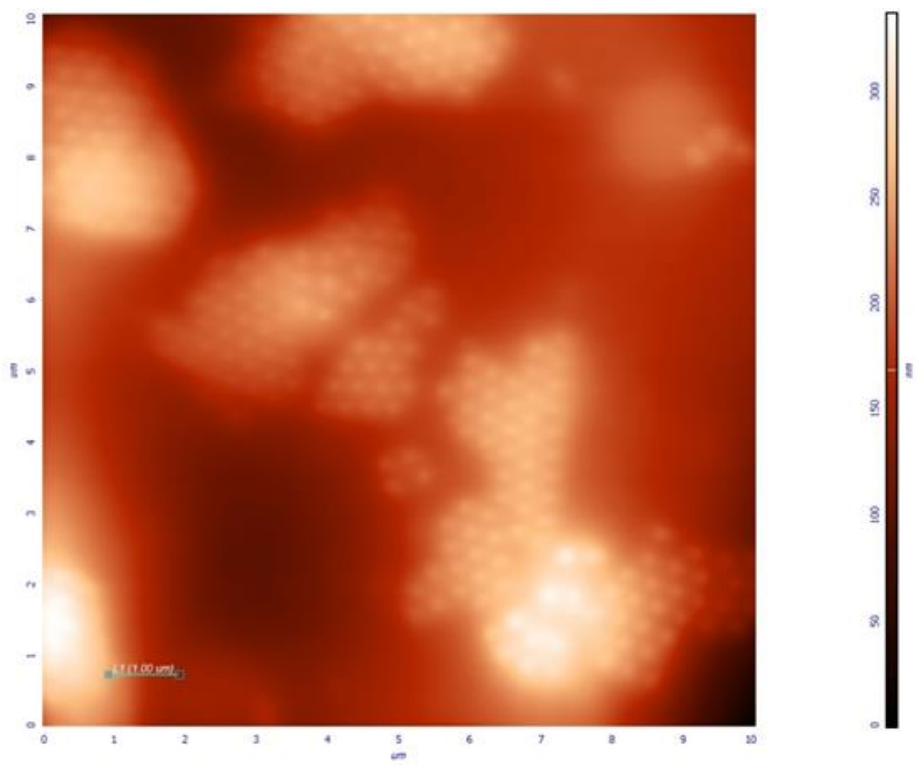

Figure S9. AFM topographic image of a film made from a PAA/latex mixture with $\mathrm{Pe}=9.9$ and $\phi_{\mathrm{pp}}=0.2 ; \phi=0.015$ for the colloid. Regions attributed to the PAA partially blanketing the colloidal surface. Island-like regions of colloids project above the surrounding area coated with PAA. Image size is $10 \mu \mathrm{m} \times 10 \mu \mathrm{m}$. 
Table S2. Dynamic Contact Angle Analysis of Water on Polymer/Colloid Films

\begin{tabular}{|l|c|c|c|l|}
\hline Sample* & Pe & $\begin{array}{c}\text { Mean } \\
\text { Advancing angle } \\
()^{\circ}\end{array}$ & $\begin{array}{c}\text { Mean } \\
\text { Receding Angle } \\
\left({ }^{\circ}\right.\end{array}$ & $\begin{array}{c}\text { Hysteresis }\left(^{\circ}\right) \\
\pm \text { SD }\end{array}$ \\
\hline Poly(acrylate) & 8.3 & 33.9 & 33.7 & $0.2 \pm 2.4$ \\
\hline dPAA & 9.9 & 57.0 & 49.7 & $7.3 \pm 4.1$ \\
\hline Poly(acrylate)/dPAA & 0.7 & 54.7 & 46.8 & $7.9 \pm 2.3$ \\
\hline Poly(acrylate)/dPAA & 2.3 & 85.9 & 62.9 & $23.0 \pm 1.4$ \\
\hline Poly(acrylate)/dPAA & 7.7 & 55.4 & 41.1 & $14.3 \pm 2.9$ \\
\hline Poly(acrylate)/dPAA & 9.9 & 95.4 & 68.2 & $27.2 \pm 7.1$ \\
\hline Poly(acrylate)/dPAA & 24.5 & 76.7 & 56.5 & $20.2 \pm 10.6$ \\
\hline Poly(acrylate)/dPAA & 25.0 & 100.7 & 79.3 & $21.4 \pm 4.0$ \\
\hline
\end{tabular}

* Analysis was performed on the same samples used in the ERD experiments.

\section{References}

(1) Reith, D.; Muller, B.; Muller-Plathe, F.; Wiegand, S. How Does the Chain Extension of Poly(Acrylic Acid) Scale in Aqueous Solution? A Combined Study with Light Scattering and Computer Simulation. J. Chem. Phys. 2002, 116 (10), 9100-9106. https://doi.org/10.1063/1.1471901.

(2) Gong, Y. H.; Shen, C.; Lu, Y. Z.; Meng, H.; Li, C. X. Viscosity and Density Measurements for Six Binary Mixtures of Water (Methanol or Ethanol) with an Ionic Liquid ([BMIM][DMP] or [EMIM][DMP]) at Atmospheric Pressure in the Temperature Range of (293.15 to 333.15) K. J. Chem. Eng. Data 2012, 57 (1), 33-39. https://doi.org/10.1021/je200600p.

(3) Sear, R. P.; Warren, P. B.; Sear, R. P. Diffusiophoresis in Non-Adsorbing Polymer Solutions: The Asakura-Oosawa Model and Stratification in Drying Films. Phys. Rev. E 2017, 96 (6), 062602. https://doi.org/10.1103/PhysRevE.96.062602.

(4) Mayer, M. SIMNRA User's Guide. Rep. IPP 1997, 9 (113).

(5) Gurbich, A. F. SigmaCalc Recent Development and Present Status of the Evaluated Cross-Sections for IBA. Nucl. Instruments Methods Phys. Res. Sect. B Beam Interact. with Mater. Atoms 2016, 371, $27-32$. https://doi.org/10.1016/j.nimb.2015.09.035.

(6) Kellock, A. J.; Baglin, J. E. E. Absolute Cross Section for D(4He, D)4He Forward Scattering. Nucl. Inst. Methods Phys. Res. B 1993, 79 (1-4), 493-497. https://doi.org/10.1016/0168-583X(93)95397-N.

(7) Prawer, S.; Nemanich, R. J. Raman Spectroscopy of Diamond and Doped Diamond. Philosophical Transactions of the Royal Society A: Mathematical, Physical and Engineering Sciences. 2004, pp 25372565. https://doi.org/10.1098/rsta.2004.1451.

(8) Dychalska, A.; Popielarski, P.; Franków, W.; Fabisiak, K.; Paprocki, K.; Szybowicz, M. Study of CVD Diamond Layers with Amorphous Carbon Admixture by Raman Scattering Spectroscopy. Mater. Sci. Pol. 2015, 33 (4), 799-805. https://doi.org/10.1515/msp-2015-0067. 